\title{
A five IncRNA signature for prognosis prediction in hepatocellular carcinoma
}

\author{
YONGQIANG SUN ${ }^{1}$, FANGFANG ZHANG $^{2}$, LIFU WANG $^{1}$, XUEAI SONG ${ }^{1}$, \\ JING JING ${ }^{1}$, FAN ZHANG ${ }^{1}$, SIMIAO YU ${ }^{1}$ and HONGHONG LIU ${ }^{3}$ \\ ${ }^{1}$ Integrative Medical Center; ${ }^{2}$ Outpatient Department; ${ }^{3}$ International Center for Liver Disease Treatment, \\ 302 Military Hospital of China, Beijing 100039, P.R. China
}

Received July 13, 2018; Accepted February 2, 2019

DOI: $10.3892 / \mathrm{mmr} .2019 .10203$

\begin{abstract}
This study was conducted to screen prognosisassociated long-noncoding RNAs (lncRNAs) and a prognosis assessment model in hepatocellular carcinoma (HCC). IncRNA- and mRNA-sequencing data of early-stage HCC samples were downloaded from The Cancer Genome Atlas database. The samples were divided into training set and validation set. Differentially expressed lncRNAs (DELs) between poor prognosis and good prognosis samples were screened with DEseq and edgeR. Cox regression analysis was conducted to identify prognosis-associated lncRNAs in the training set. A prognosis risk assessment model was established to calculate the risk score for each patient in the training set, and the prognosis prediction function was tested and validated in the validation dataset. The connection between the risk assessment model and clinical features was analyzed. A co-expression network between lncRNAs and corresponding genes was constructed, and functional enrichment was performed for these genes. A total of 81 DELs were screened between poor and good prognosis samples in the training set, and 43 prognosis-associated lncRNAs were observed. Of these DELs, five were used to construct the risk assessment model (RP11-325L7.2, DKFZP434L187, RP11-100L22.4, DLX2-AS1 and RP11-104L21.3). Low-risk samples exhibited longer survival time compared with the high-risk samples. The five lncRNAs exhibited significant differences in expression levels between different prognosis groups. Risk score was an
\end{abstract}

Correspondence to: Dr Honghong Liu, International Center for Liver Disease Treatment, 302 Military Hospital of China, 100 West Fourth Ring Road, Fengtai, Beijing 100039, P.R. China

E-mail: liuhonghong302@126.com

Abbreviations: HCC, hepatocellular carcinoma; lncRNA, long noncoding RNA; DEL, differentially expressed lncRNA; PPAR, peroxisome proliferator-activated receptor; RFS, recurrence-free survival; ROC, receiver operating characteristic

Key words: HCC, DELs, risk assessment model, co-expression network, prognosis independent prognostic factor for HCC. In the entire set, the low-risk group demonstrated significantly better prognosis compared with the high-risk group, even across all age, sex and alcohol consumption subgroups. 'Nucleoside-triphosphatase regulator activity', 'GTPase regulator activity', 'enzyme binding', 'peroxisome proliferator-activated receptor signaling pathway' and 'fatty acid metabolism' were the most significantly enriched functional terms. The signature lncRNAs screened in this study may have constitute novel strategies and biomarkers that predict the prognosis of HCC, and these may also contribute to a deeper understanding of the mechanisms underlying HCC development.

\section{Introduction}

Hepatocellular carcinoma (HCC), a primary malignancy of the liver, predominantly occurs in patients with chronic liver disease and cirrhosis. HCC is the most common type of primary liver cancer in adults, and the most common cause of mortality in patients with cirrhosis (1). An estimated 662,000 $\mathrm{HCC}$-associated mortalities are reported annually worldwide, and half of these cases have occurred in China (2).

The prognosis of HCC is poor, as only $10-20 \%$ carcinomas can be completely removed by surgery. The patients usually succumb to the disease within 3-6 months (3). With the approval of sorafenib for advanced HCC treatment, the prognosis of metastatic or unresectable HCC has improved (4). However, more advanced and efficient therapies, and better prognostic molecular markers are required.

Long-noncoding RNAs (IncRNAs), accounting for $80 \%$ of noncoding RNAs, are defined as endogenous cellular RNAs longer than 200 nucleotides that lack an obvious open-reading frame (5). The role of lncRNAs in the carcinogenesis, microvascular invasion and metastasis of HCCs has been reported in a number of studies (6-8). For instance, the overexpression of lncRNA-urothelial cancer associated 1 contributed to HCC progression through the inhibition of miR-216b, and subsequent activation of the fibroblast growth factor receptor 1/extracellular signal-regulated kinase signaling pathway (9). lncRNA HOX transcript antisense RNA (HOTAIR) overexpression was demonstrated to predict tumor recurrence in patients with HCC following liver transplantation (3). Furthermore, upregulation of lncRNA ZEB1-antisense RNA 1 (AS1) and 
ANRIL expression was reported to predict a poorer prognosis in HCC $(10,11)$. In comparison with HCC patients without HOTAIR expression, those with high HOTAIR expression exhibited significantly poorer prognosis (12). Downregulation of lncRNA growth arrest specific 5 expression was also associated with HCC prognosis (13). Despite these studies, the functions of multiple lncRNAs in HCC remain unclear. Therefore, a comprehensive analysis of HCC-associated lncRNAs is necessary in order to reveal possible biomarkers and/or potential therapeutic targets.

In the present study, RNA-sequencing (RNA-seq) data of HCC samples were studied to select signature lncRNAs for the prognosis of HCC. Differentially expressed lncRNAs (DELs) were identified, and prognosis-associated lncRNAs were screened. Based on a risk score calculated using prognosis-linked lncRNAs, a risk assessment model was established. This model was tested by performing a survival analysis of high-risk and low-risk samples from the validation and entire datasets. Multivariate and univariate analyses were performed to investigate prognosis-associated clinical factors. Moreover, the genes associated with signature lncRNAs were screened for co-expression network and functional enrichment analyses.

\section{Materials and methods}

Data acquisition and processing. The RNA-seq data of HCC samples were downloaded from The Cancer Genome Atlas database (https://gdc-portal.nci.nih.gov/; May 8, 2017). A total of 424 samples were analyzed based on Illumina HiSeq 2000 RNA Sequencing platform. Of these, 261 samples from early stage (stage I and II) cancer were used in the present study. Among these samples, 194 samples with available clinical information were retained, and the samples with <6-month recurrence-free survival (RFS) time and the non-recurrent samples were discarded. Hence, 167 samples remained for further analyses, and these were randomly divided into training (83 samples) and validation datasets (84 samples).

Screening of DELs. The 83 training samples were divided into poor prognosis samples (recurrence samples with RFS $<24$ months) and good prognosis samples (non-recurrent samples with RFS >24 months). DEseq (14) and edgeR (15) packages in $\mathrm{R}$ (version 3.1.0; R-project.org/) were used for the screening of the DELs between poor and good prognosis samples. The threshold was set as false discovery rate $<0.05$ and $\mid \log ($ fold change $) \mid>1.3$. Overlapping lncRNAs screened using the two packages were used for further analyses.

Screening of prognosis-associated lncRNAs. Cox regression analysis was conducted for the identification of prognosis-associated lncRNAs in the training set using the survival package in $\mathrm{R}$ (16). The significance of the identified lncRNAs was tested with log-rank test, and $\mathrm{P}<0.05$ was considered to indicate a statistically significant difference.

Construction of a risk assessment model. The prognostic risk of each sample in the training set was calculated using the regression coefficients of prognosis-associated lncRNAs via multivariate Cox regression analysis. The following formula was used for calculation, wherein the regression coefficient of each lncRNA was weighted:

$$
\begin{aligned}
& \text { Risk score }=\beta \text { lncRNA1 } x \operatorname{expr}_{\text {IncRNAl }}+\beta \operatorname{lncRNA2} x \\
& \operatorname{expr}_{\operatorname{lncRNA2} 2}+\cdots+\beta \operatorname{lncRNAn} x \operatorname{expr}_{\text {IncRNAn }}
\end{aligned}
$$

where, $\beta$ lncRNAn indicates the multivariate Cox regression coefficient and $\operatorname{expr}_{\text {IncRNAn }}$ indicates the expression level of lncRNAn.

Association between risk assessment model and clinical feature. The obtained formula was used to calculate the risk score of patients in the validation set. The median risk score was used to distinguish between high-risk and low-risk samples. The survival information was compared using Kaplan-Meier survival analysis and receiver operating characteristic (ROC) curve (17). The area under the ROC curve (AUC) indicated the accuracy of prognosis. An AUC $>0.5$ indicated a relatively high accuracy of prognosis, with higher AUC values indicating a more accurate prognosis. The high-risk and low-risk samples were also subjected to Cox regression analysis to screen for prognosis risk-associated clinical features. The independent prognosis factors were identified using both univariate and multivariate Cox regression analyses. Moreover, the association between high or low risk and prognosis under the same clinical status was tested.

Co-expression network and functional enrichment. The co-expression network between lncRNAs and the corresponding mRNAs was constructed using the MEM package $(18,19)$. $\mathrm{P}<0.05$ was used as the cut-off selection criterion. Gene pairs associated with lncRNAs were obtained from the Search Tool for the Retrieval of Interacting Genes/Proteins database (string-db.org/), using a connection score $>0.4$ as threshold. A co-expression network of these genes was also constructed. Functional enrichment of these lncRNA-associated genes was conducted using the Database for Annotation, Visualization and Integrated Discovery (david.ncifcrf.gov/), and $\mathrm{P}<0.05$ was set as the cut-off value (20).

\section{Results}

Identification and validation of five lncRNA prognostic signatures in two datasets. The clinical information of the samples in the training set, validation set and entire set is presented in Table I. A total of 23 poor prognosis samples and 18 good prognosis samples were reported in the training set. Using edgeR and DEseq packages for R, 117 and 225 DELs were screened comparing the poor and good prognosis samples, respectively. The bidirectional hierarchical clustering results of the overlapped 81 lncRNAs are displayed in Fig. 1.

A total of 43 prognosis-associated lncRNAs were obtained using univariate Cox regression analysis. Five of these IncRNAs were selected for multivariate Cox regression analysis to construct the risk assessment model. These five signature lncRNAs were RP11-325L7.2, DKFZP434L187, RP11-100L22.4, DLX2-AS1 and RP11-104L21.3 (Table II).

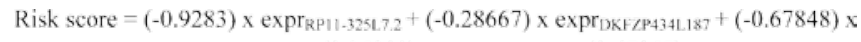
$\operatorname{expr}_{\mathrm{RP} 11-1001.22 .4}+(0.35902) \mathrm{x} \operatorname{expr}_{\mathrm{DL} X 2-\mathrm{AS} 1}+(0.67857) \times \operatorname{expr}_{\mathrm{RP} 11-1041.21 .3}$ 
Table I. Clinical information of samples in training set, validation set and entire set.

Stage I + II

Clinical parameters

$\begin{array}{ccc}\text { Training set }(\mathrm{n}=83) & \text { Testing set }(\mathrm{n}=84) & \text { Entire set }(\mathrm{n}=167) \\ 58.99 \pm 11.82 & 58.42 \pm 13.35 & 59.14 \pm 12.59 \\ 61 / 22 & 56 / 28 & 117 / 50 \\ 64 / 19 & 70 / 14 & 134 / 33 \\ 61 / 22 & 63 / 21 & 124 / 43 \\ 55 / 28 & 58 / 26 & 113 / 54 \\ 14 / 5 / 14 / 50 & 10 / 4 / 8 / 62 & 24 / 9 / 22 / 112 \\ 29 / 47 / 7 & 22 / 59 / 3 & 51 / 106 / 10 \\ 31 / 52 & 32 / 52 & 63 / 104 \\ 24.07 \pm 20.45 & 24.56 \pm 21.41 & 24.32 \pm 20.87\end{array}$

Age (years, mean $\pm \mathrm{sd})$

Gender (male/female)

Pathologic M (M0/-)

Pathologic N (NO/-)

Pathologic T (T1/T2)

Virus infection (HBV/HCV/mixed/non)

Alcohol consumption (yes/no/-)

Recurrence (yes/no)

Recurrence free survival time (months, mean \pm sd)

$24.07 \pm 20.45$

$24.56 \pm 21.41$

sd, standard deviation; -, data unavailable; HBV, hepatitis B virus; $\mathrm{HCV}$, hepatitis C virus.

Table II. lncRNAs in the risk assessment model.

\begin{tabular}{lccccr}
\hline IncRNA & Coefficient & HR & Lower .95 & Upper .95 & P-value \\
\hline RP11-325L7.2 & -0.9283 & 0.3952 & 0.2193 & 0.7124 & 0.002 \\
DKFZP434L187 & -0.2867 & 0.7508 & 0.6238 & 0.9036 & 0.002 \\
RP11-100L22.4 & -0.6785 & 0.5074 & 0.2808 & 0.9167 & 0.025 \\
DLX2-AS1 & 0.3590 & 1.4319 & 1.0420 & 1.9678 & 0.027 \\
RP11-104L21.3 & 0.6786 & 1.9711 & 1.0793 & 3.5996 & 0.027 \\
\hline
\end{tabular}

lncRNA, long noncoding RNA; HR, hazard ratio.
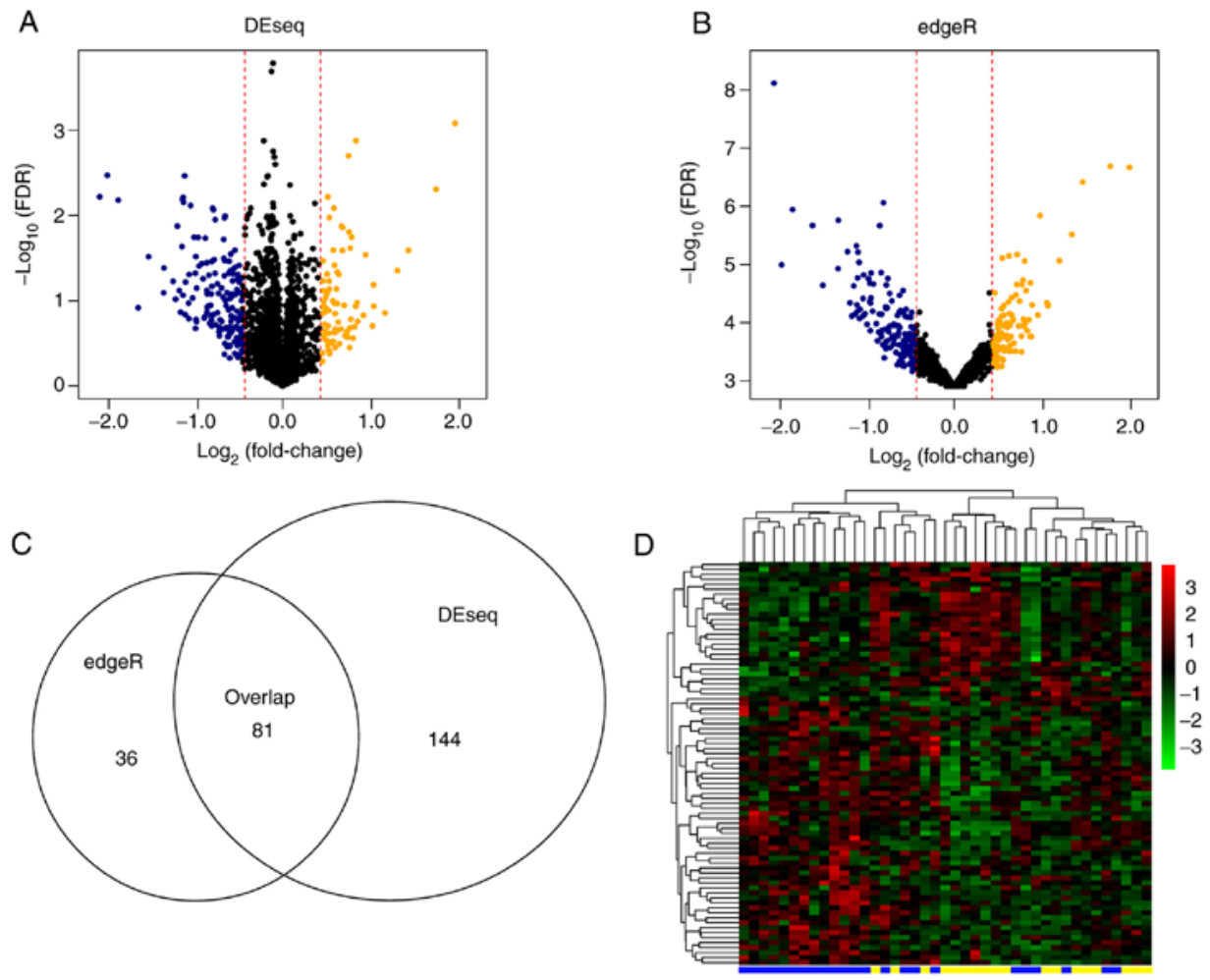

Figure 1. DEL analysis. (A) DELs identified using the DESeq package. (B) DELs identified using the edgeR package. (C) Overlapping DELs between the selection methods. (D) Heatmap of the 81 common DELs in the good and poor prognosis groups. Blue represents bad prognosis sample, while yellow indicates good prognosis sample. DEL, differentially expressed long noncoding RNA; FDR, false discovery rate. 

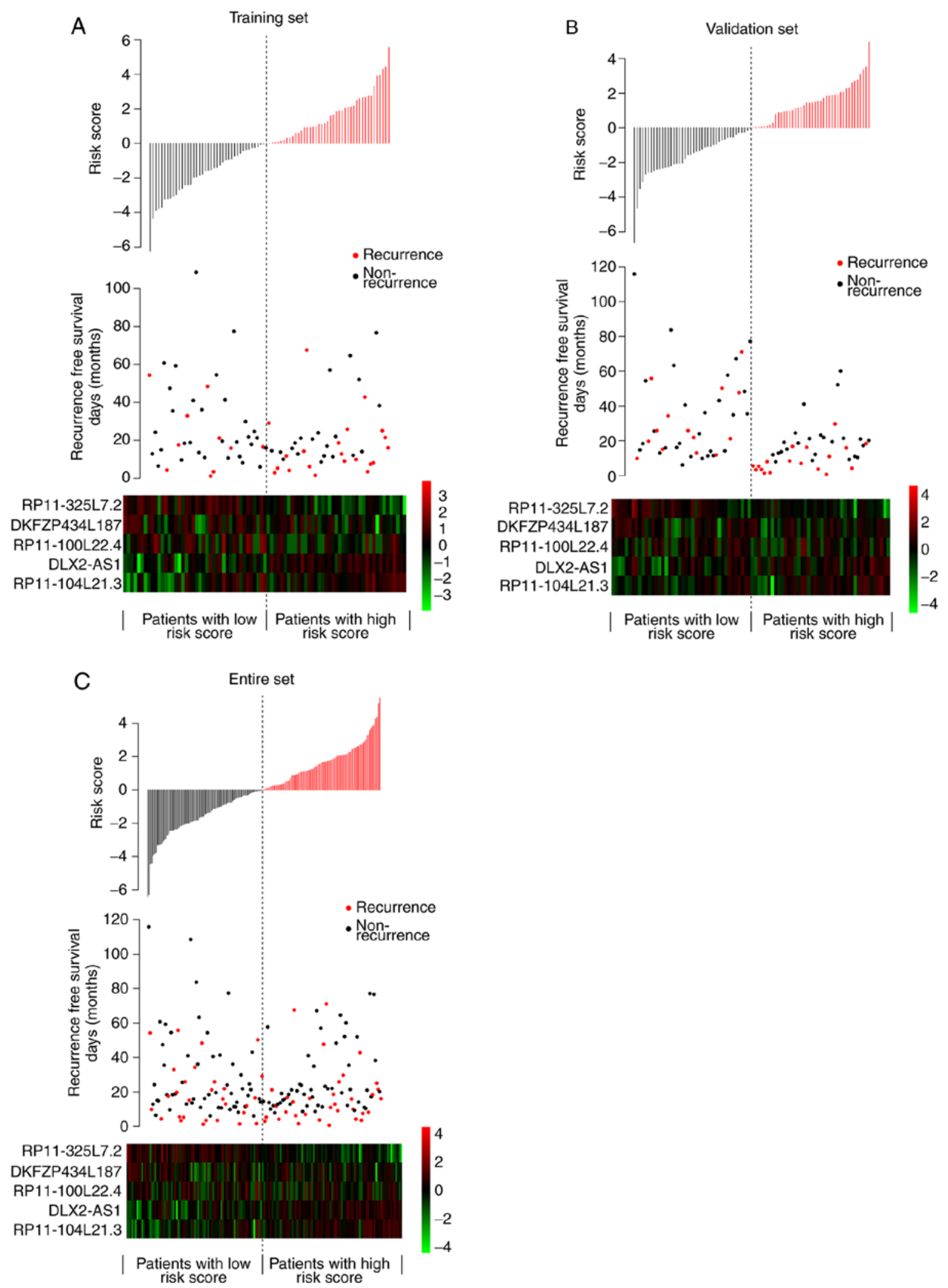

Figure 2. Risk score distributions, RFS status, and expressions of five signature lncRNAs in three datasets. Risk score distributions, RFS status, and expressions of IncRNAs in the (A) training set, (B) validation set and (C) entire set. In the risk score distribution graphs, the horizontal axis represents samples categorized between low and high-risk scores, while the vertical axis represents risk scores. In the RFS status graphs, the horizontal axis represents samples with low to high-risk scores, while vertical axis represents RFS time. Recurrence samples and non-recurrence samples are marked in red and black, respectively. RFS, recurrence-free survival; lncRNAs, long noncoding RNAs.

These five lncRNAs were used to evaluate the HCC risk in each patient. The risk score distribution, RFS status and expression of five signature IncRNAs in training set, validation set and entire set are depicted in Fig. 2. Their distributions were similar, supporting the robust prediction ability of the five lncRNA-based risk score assessment model. 

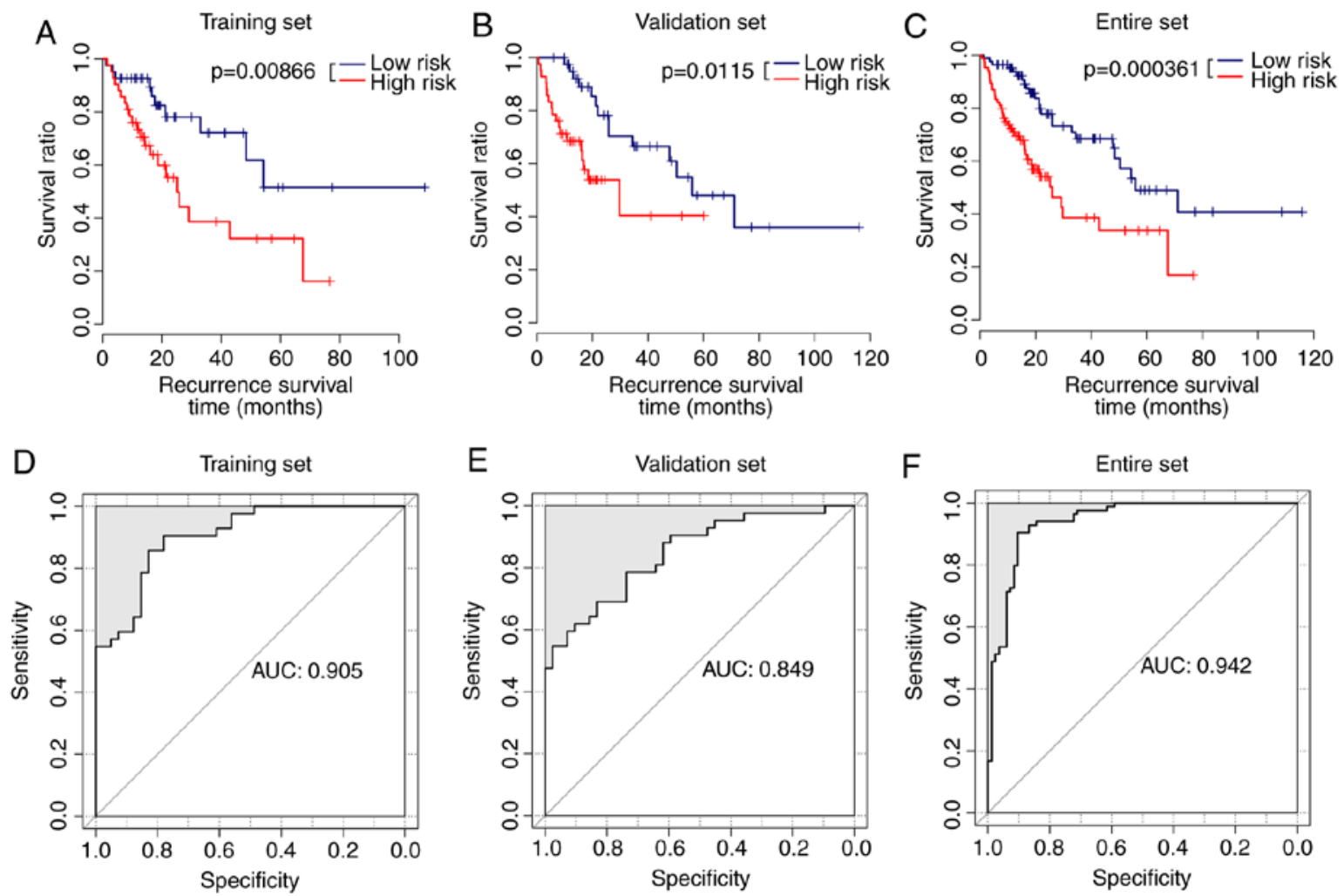

Figure 3. Kaplan-Meier curves of recurrence free survival and receiver operating characteristic for the five long noncoding RNAs. Kaplan-Meier curves in training set (A), validation set (B) and entire set (C). Horizontal axis represents the survival period and the vertical axis represents the frequency. Red lines represent high-risk group samples, blue lines represent low-risk group samples. Receiver operating characteristic curves in training set (D), validation set (E) and entire set (F). The abscissa represents sensitivity and the ordinate represents specificity. AUC, area under the curve.
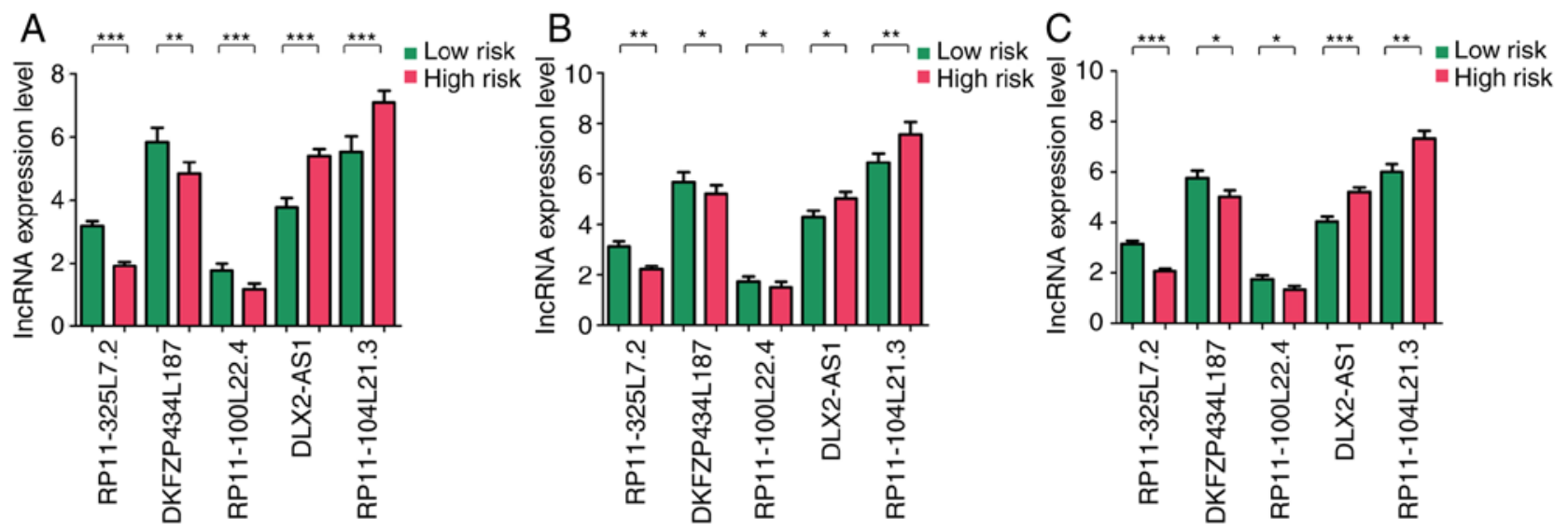

Figure 4. Expression level comparisons of the five signature lncRNAs in high- and low-risk samples across the three datasets. Expression levels in (A) training set, (B) validation set and (C) entire set. The expression level in low-risk samples is displayed in green, while the expression level in high-risk samples is displayed in red. ${ }^{*} \mathrm{P}<0.05,{ }^{* *} \mathrm{P}<0.01$ and ${ }^{* * *} \mathrm{P}<0.005$. IncRNA, long noncoding RNA.

Survival analysis using the five lncRNAs in the training, validation and entire sets. Using the risk assessment model, samples in the training set were divided into high-risk group (42 samples) and low-risk group (41 samples). The Kaplan-Meier survival curves indicated that the samples from the low-risk group had longer survival times compared with those from the high-risk group (average RFS was $27.22 \pm 22.23$ vs. $21.01 \pm 18.28$ months) in the training set (Fig. 3A). Similar results were observed for the samples from the validation set. Samples from the low-risk group had longer survival times compared with those from the high-risk group (average RFS was 33.50 \pm 24.67 vs. $15.62 \pm 12.45$ months; Fig. 3B). The samples from the low-risk group similarly exhibited longer survival times compared with those from the high-risk group (average RFS was 30.39 \pm 23.57 vs. $18.31 \pm 5.78$ months; Fig. $3 \mathrm{C}$ ) in the entire set. The average AUC value of these five lncRNAs in the training set was 0.905 (Fig. 3D), suggestive of the highly accurate prognosis using these five lncRNAs. The average AUC value of these five lncRNAs in the validation set was 0.849 (Fig. 3E). The average AUC value of the lncRNAs for the entire dataset was 0.942 (Fig. 3F). 

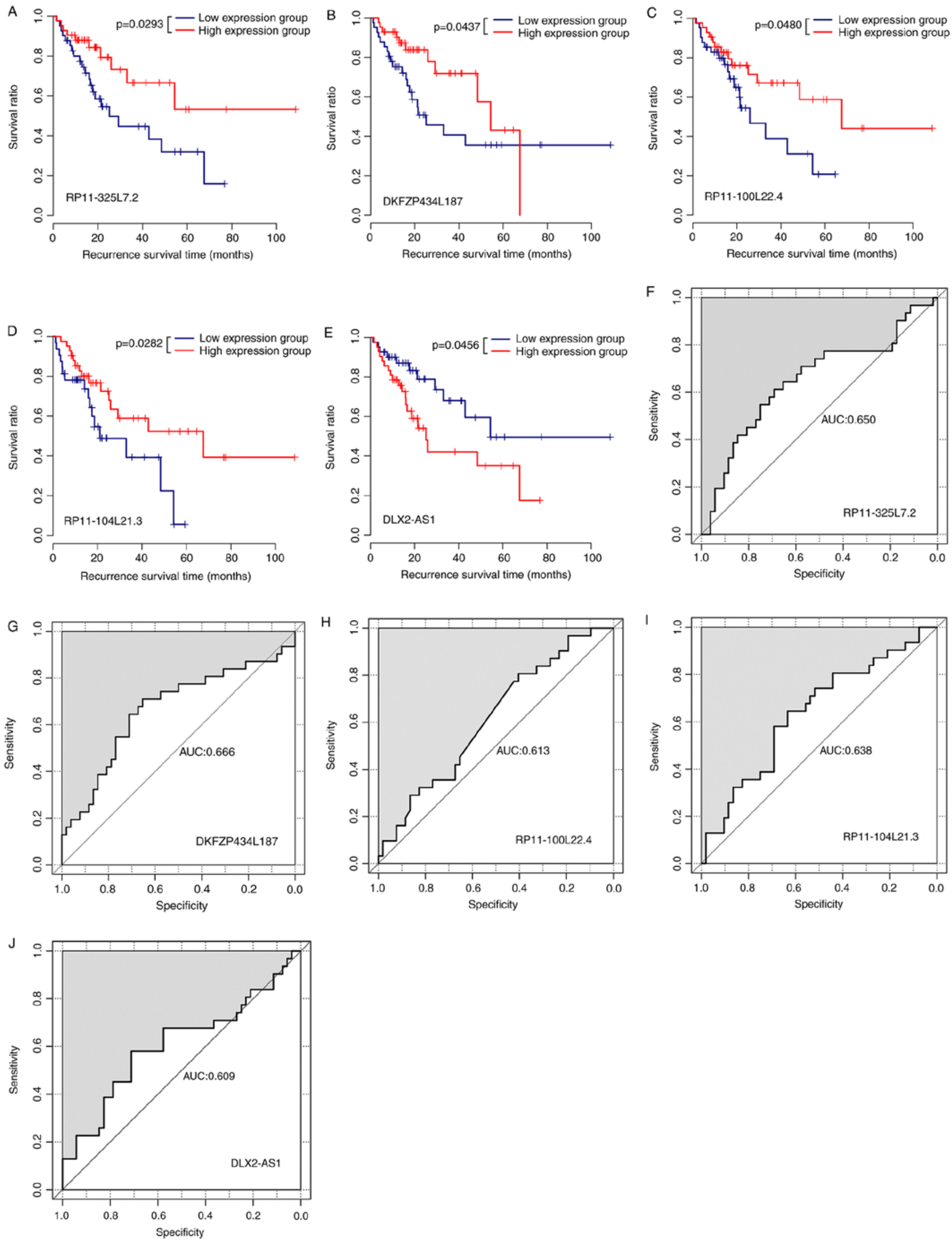

Figure 5. Predictive effect of each signature lncRNA for high-risk and low-risk samples in the validation set. Kaplan-Meier survival curves for (A) RP11-325L7.2, (B) DKFZP434L187, (C) RP11-100L22.4, (D) RP11-104L21.3 and (E) DLX2-AS1 lncRNAs. High-risk and low-risk samples are marked in red and blue, respectively. Receiver operating characteristic curves for (F) RP11-325L7.2, (G) DKFZP434L187, (H) RP11-100L22.4, (I) DLX2-AS1 and (J) RP11-104L21.3 lncRNAs. IncRNA, long noncoding RNA; AUC, area under the curve. 
Table III. Cox regression results between clinical features and prognosis.

A, Training set $(n=83)$

\begin{tabular}{|c|c|c|c|c|c|c|}
\hline \multirow[b]{2}{*}{ Variables } & \multicolumn{3}{|c|}{ Univariate analysis } & \multicolumn{3}{|c|}{ Multivariate analysis } \\
\hline & HR & $95 \% \mathrm{CI}$ & P-value & HR & $95 \% \mathrm{CI}$ & P-value \\
\hline Risk score (high/low) & 2.557 & $1.200-5.447$ & 0.009 & 2.452 & $0.544-3.048$ & 0.024 \\
\hline Age $(\leq 60 />60)$ & 0.943 & $0.464-1.914$ & 0.869 & 2.172 & $0.526-3.964$ & 0.283 \\
\hline Gender (male/female) & 0.938 & $0.429-2.050$ & 0.872 & 0.528 & $0.495-1.291$ & 0.265 \\
\hline Virus infection (HBV/HCV/mixed) & 1.317 & $0.723-2.398$ & 0.369 & 1.283 & $0.653-2.519$ & 0.469 \\
\hline Alcohol consumption (yes/no) & 1.402 & $0.752-1.963$ & 0.046 & 1.103 & $0.713-1.933$ & 0.043 \\
\hline
\end{tabular}

B, Validation set $(n=84)$

Univariate analysis

\begin{tabular}{|c|c|c|c|c|c|c|}
\hline \multirow[b]{2}{*}{ Variables } & \multicolumn{3}{|c|}{ Univariate analysis } & \multicolumn{3}{|c|}{ Multivariate analysis } \\
\hline & HR & $95 \% \mathrm{CI}$ & $\mathrm{P}$-value & HR & $95 \% \mathrm{CI}$ & P-value \\
\hline Risk score (high/low) & 2.723 & $1.289-5.751$ & 0.012 & 2.992 & $1.196-3.458$ & 0.034 \\
\hline Age $(\leq 60 />60)$ & 1.107 & $0.547-2.241$ & 0.777 & 0.265 & $0.040-1.767$ & 0.170 \\
\hline Gender (male/female) & 0.645 & $0.319-1.300$ & 0.216 & 0.561 & $0.090-3.522$ & 0.538 \\
\hline Virus infection (HBV/HCV/mixed) & 1.098 & $0.467-2.059$ & 0.958 & 2.243 & $0.678-7.430$ & 0.186 \\
\hline Alcohol consumption (yes/no) & 1.132 & $0.298-1.802$ & 0.495 & 1.358 & $0.520-2.466$ & 0.296 \\
\hline
\end{tabular}

C, Entire set $(n=167)$

\begin{tabular}{|c|c|c|c|c|c|c|}
\hline \multirow[b]{2}{*}{ Variables } & \multicolumn{3}{|c|}{ Univariate analysis } & \multicolumn{3}{|c|}{ Multivariate analysis } \\
\hline & HR & $95 \% \mathrm{CI}$ & $\mathrm{P}$-value & HR & $95 \% \mathrm{CI}$ & $\mathrm{P}$-value \\
\hline Risk score (high/low) & 2.569 & $1.530-4.315$ & 0.001 & 1.906 & $0.697-5.205$ & 0.021 \\
\hline Age $(\leq 60 />60)$ & 1.018 & $0.619-1.672$ & 0.944 & 0.215 & $0.437-3.518$ & 0.686 \\
\hline Gender (male/female) & 0.773 & $0.462-1.294$ & 0.328 & 0.424 & $0.566-4.132$ & 0.402 \\
\hline Virus infection (HBV/HCV/mixed) & 1.178 & $0.746-1.860$ & 0.481 & 1.180 & $0.715-1.948$ & 0.517 \\
\hline Alcohol consumption (yes/no) & 1.542 & $0.803-1.648$ & 0.047 & 1.209 & $0.554-1.792$ & 0.021 \\
\hline
\end{tabular}

$\mathrm{HR}$, hazard ratio; $\mathrm{CI}$, confidence interval; $\mathrm{HBV}$, hepatitis $\mathrm{B}$ virus; $\mathrm{HCV}$, hepatitis $\mathrm{C}$ virus.

Significant differences were observed in the expression levels of these five lncRNAs between samples from the lowand high-risk groups in training set, validation set and entire set $(\mathrm{P}<0.05$; Fig. 4A-C, respectively). Expression levels of DLX2-AS1 and RP11-104L21.3 were significantly higher in the samples from the high-risk group compared with those from the low-risk group, whereas the expression levels of DKFZP434L187, RP11-100L22.4 and RP11-325L7.2 were significantly lower in the samples from the high-risk group compared with those from the low-risk group $(\mathrm{P}<0.05)$.

Association between survival information and expression pattern was different for the five lncRNAs. In the validation set, high expression samples had a longer survival time compared with the low expression group for RP11-325L7.2, DKFZP434L187, RP11-100L22.4 and RP11-104L21.3 (Fig. 5A-D, respectively), suggesting that the high expression of these lncRNAs may be associated with good prognosis. Low DLX2-AS1 expression was associated with longer survival time compared with those from the high expression group (Fig. 5E), indicating that high expression of DLX2-AS1 may be associated with poor prognosis. The AUC values for prognosis using RP11-325L7.2, DKFZP434L187, RP11-100L22.4 and RP11-104L21.3 (Fig. 5F-I, respectively) were $0.650,0.666,0.613$, and 0.638 , respectively, indicating that these four lncRNAs demonstrated relatively high accuracy for prognosis, and the best performance was produced by DKFZP434L187. The AUC value for DLX2-AS1 prognosis was 0.609 (Fig. 5J), indicative of the relatively and equally high accuracy of prognosis using this lncRNA.

Prognostic factors for HCC. The results of univariate and multivariate Cox regression analyses are presented in Table III. In the training, validation and entire sets, the risk score was 
A Younger dataset $($ age $<=60, \mathrm{~N}=83)$

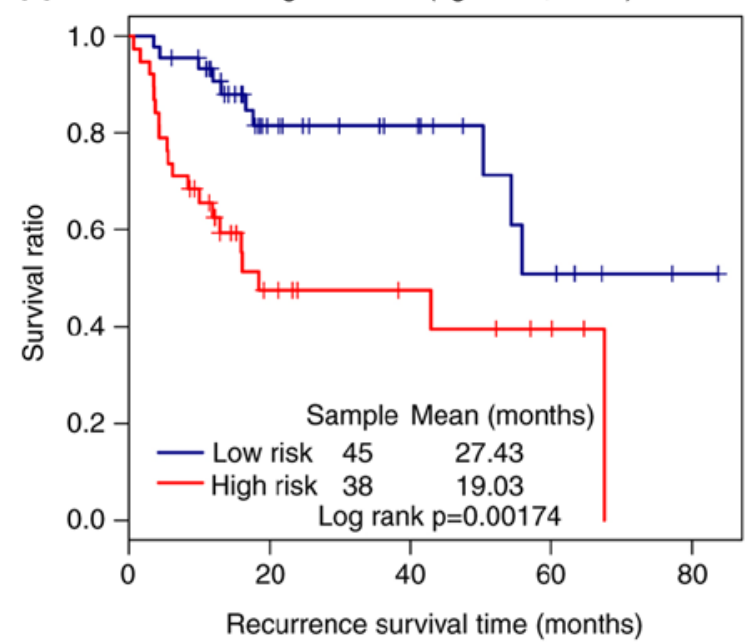

B

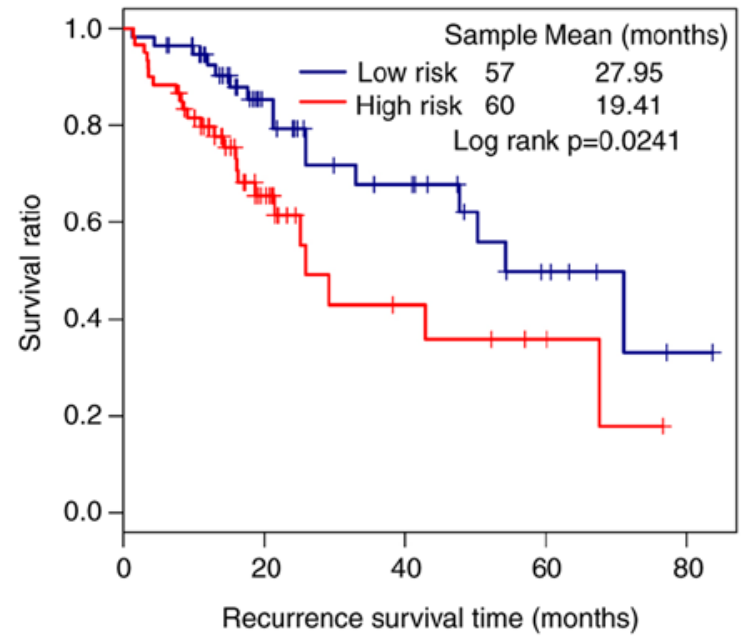

C

Alcohol dataset $(\mathrm{N}=51)$

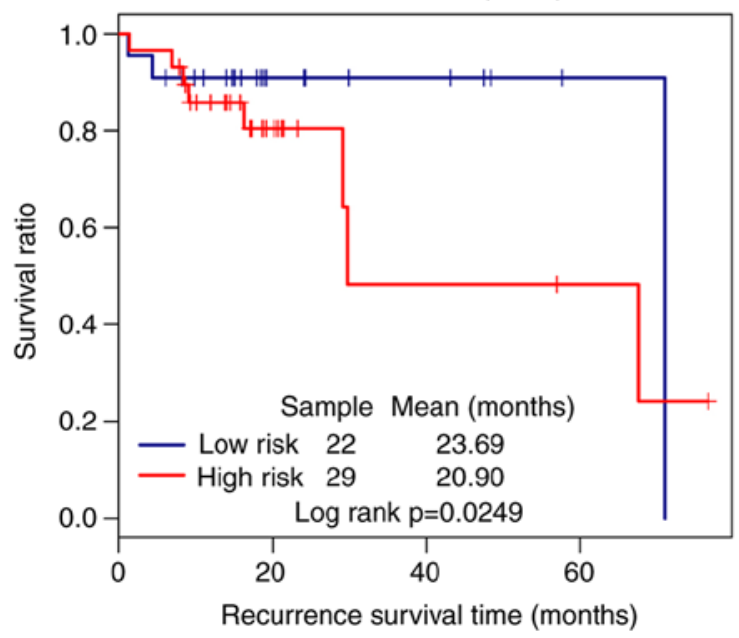

Elder dataset (age $>60, \mathrm{~N}=84$ )

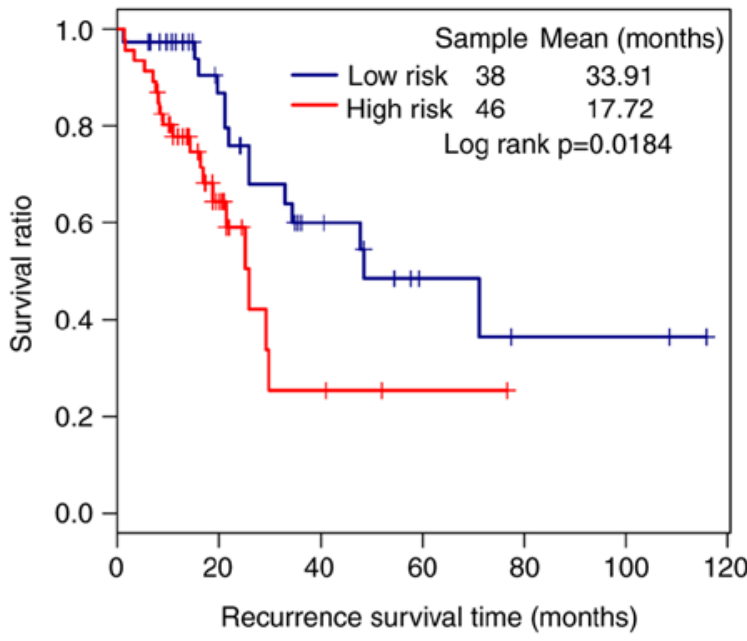

Female dataset $(\mathrm{N}=50)$
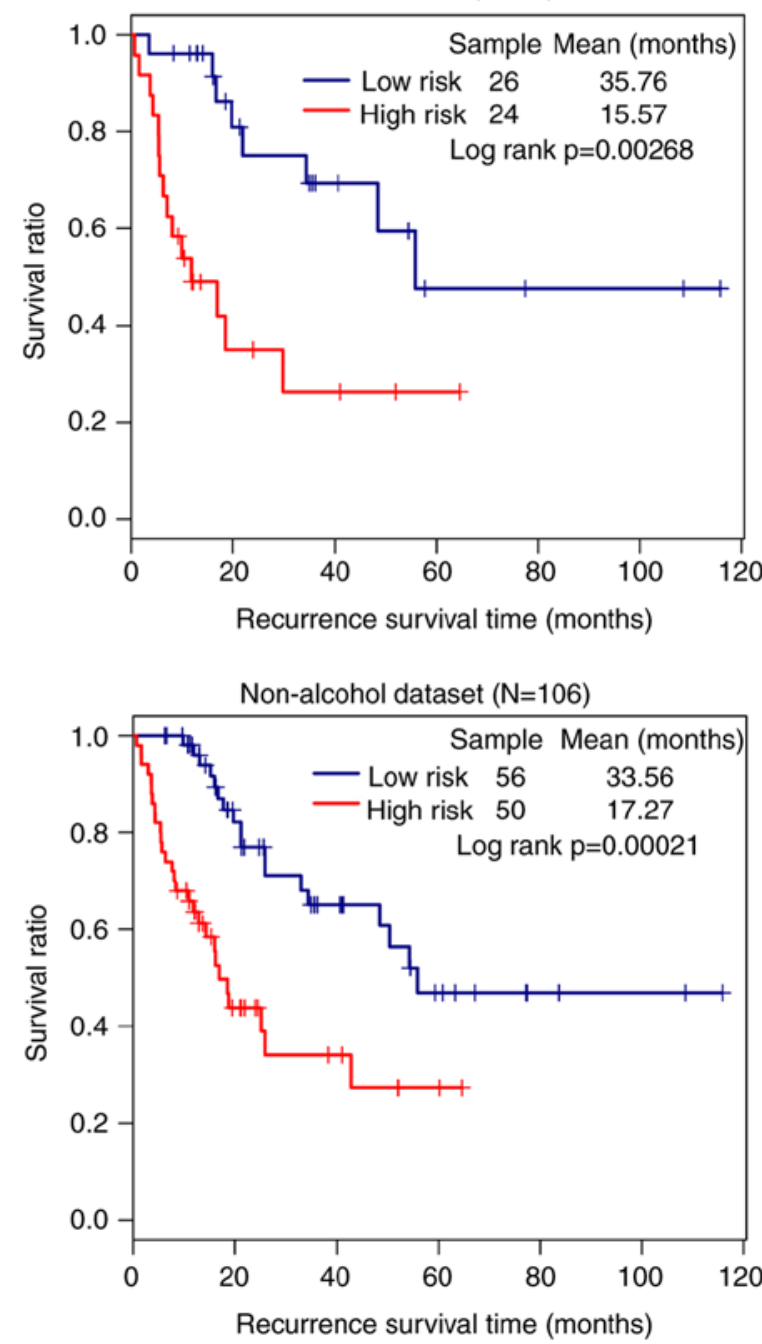

Figure 6. Kaplan-Meier curves of recurrence free survival between high- and low-risk patients across the entire dataset. Survival across the entire dataset was divided and evaluated between (A) age, (B) sex and (C) alcohol assumption subgroups. High-risk and low-risk samples are marked in red and blue, respectively.

significantly associated with the prognosis of patients and served as an independent prognostic factor $(\mathrm{P}<0.05)$. Alcohol consumption was also an independent predictive index in training and entire sets $(\mathrm{P}<0.05)$. Virus infection was not a significant factor $(\mathrm{P}>0.05)$.
The impact of high and low risk on prognosis within subgroups of the same clinical feature was evaluated. In the entire set, the low-risk group had a significantly better prognosis compared with the high-risk group in all age, sex and alcohol subgroups ( $\mathrm{P}<0.05$; Fig. 6A-C, respectively). 
Table IV. Significantly enriched functions and pathways of genes correlated with signature long noncoding RNAs.

A, Enriched functions (GO terms)

\begin{tabular}{llrr}
\hline Category & \multicolumn{1}{c}{ Term } & Gene count & P-value \\
\hline GOTERM_MF_FAT & GO: 0060589 nucleoside-triphosphatase regulator activity & 24 & $3.52 \times 10^{-4}$ \\
GOTERM_MF_FAT & GO: 0030695 GTPase regulator activity & 23 & $6.35 \times 10^{-4}$ \\
GOTERM_BP_FAT & GO: 0055114 oxidation reduction & 28 & $2.81 \times 10^{-3}$ \\
GOTERM_BP_FAT & GO: 0006468 protein amino acid phosphorylation & 28 & $4.93 \times 10^{-3}$ \\
GOTERM_MF_FAT & GO: 0019899 enzyme binding & 26 & $1.79 \times 10^{-3}$ \\
\hline
\end{tabular}

B, Enriched pathways (KEGG analysis)

\begin{tabular}{|c|c|c|c|}
\hline Category & Term & Gene count & P-value \\
\hline KEGG_PATHWAY & hsa03320: PPAR signaling pathway & 9 & $7.24 \times 10^{-4}$ \\
\hline KEGG_PATHWAY & hsa00071: Fatty acid metabolism & 7 & $8.66 \times 10^{-4}$ \\
\hline KEGG_PATHWAY & hsa04666: Fc- $\gamma$-R-mediated phagocytosis & 9 & $5.62 \times 10^{-3}$ \\
\hline KEGG_PATHWAY & hsa04670: Leukocyte transendothelial migration & 10 & $6.48 \times 10^{-3}$ \\
\hline KEGG_PATHWAY & hsa04920: Adipocytokine signaling pathway & 7 & $1.20 \times 10^{-2}$ \\
\hline
\end{tabular}

GO, gene ontology; KEGG, Kyoto encyclopedia of genes and genomes; GTPase, guanosine triphosphatase; PPAR, peroxisome proliferator-activated receptor; $\mathrm{MF}$, molecular function; $\mathrm{BP}$, biological process.

Functional enrichment for mRNAs co-expressed with signature lncRNAs. The top 100 mRNAs associated with the five signature lncRNAs were determined for the construction of signature lncRNA-mRNA co-expression networks, and gene-gene co-expression networks of the genes connected with signature lncRNAs (Fig. 7). WD repeat domain 5B (WDR5B) was determined to be a target gene of RP11-100L22.4 and RP11-104L21.3 lncRNAs. Functional enrichment analysis demonstrated that 'nucleoside-triphosphatase regulator activity', 'GTPase regulator activity', 'enzyme binding', 'oxidation-reduction' and 'protein amino acid phosphorylation' were the most significantly enriched functions, while 'peroxisome proliferator-activated receptor (PPAR) signaling pathway', 'fatty acid metabolism', 'Fc- $\gamma$-R-mediated phagocytosis', 'leukocyte transendothelial migration' and 'adipocytokine signaling pathway' were the most significantly enriched pathways (Table IV).

\section{Discussion}

In the present study, a series of complex bioinformatics analyses identified five lncRNAs associated with HCC prognosis, namely RP11-325L7.2, DKFZP434L187, RP11-100L22.4, DLX2-AS1 and RP11-104L21.3. A risk-model comprising these five lncRNAs was able to distinguish low-risk and high-risk samples with a relatively high prognosis accuracy. The evaluation of the target genes of these lncRNAs further revealed that 'nucleoside-triphosphatase regulator activity', 'GTPase regulator activity', 'enzyme binding', 'PPAR signaling pathway' and 'fatty acid metabolism' were potentially the most affected pathways.
To the best of our knowledge, no studies have yet reported a direct correlation between RP11-325L7.2, RP11-100L22.4 or RP11-104L21.3 expression and HCC prognosis. Other RP11-associated lncRNAs have been reported to be implicated in HCC. For instance, high expression levels of RP11-589N15.2, RP11-343N15.5 and RP11-479G22.8 were reported to be associated with the malignant phenotypes of HCC (21). In addition, RP11.404P21.3 and RP11.488L18.10 were identified as two prognostic lncRNA biomarkers for HCC (22). The lncRNA RP11-513I15.6, a member of the three exosomal RNA-based panel, has high sensitivity and specificity in the identification of HCC cases among patients with chronic hepatitis $\mathrm{C}$ virus infection and healthy individuals (23). In the present study, the samples with higher expression of the three RP11-associated lncRNAs exhibited longer survival times compared with those which had lower expression, indicating that the high expression of these lncRNAs may be associated with a good HCC prognosis.

DLX2-AS1 is also termed DLX2 divergent transcript. To the best of our knowledge, no studies have confirmed a link between this lncRNA and HCC prognosis. However, the expression of distal-less homeobox 2 (DLX2) protein has been linked with HCC. In a previous study evaluating the response of HCC cells to Actinidia chinensis root (a traditional Chinese medicine) treatment, treatment with acRoots inhibited proliferation, invasion and migration, clonality and epithelial-to-mesenchymal transition, and promoted HCC cells apoptosis by downregulating DLX2 expression (24). Moreover, high expression level of DLX2 was linked to poor prognosis in patients with HCC (24). Another study revealed the association between DLX2 overexpression and poor 

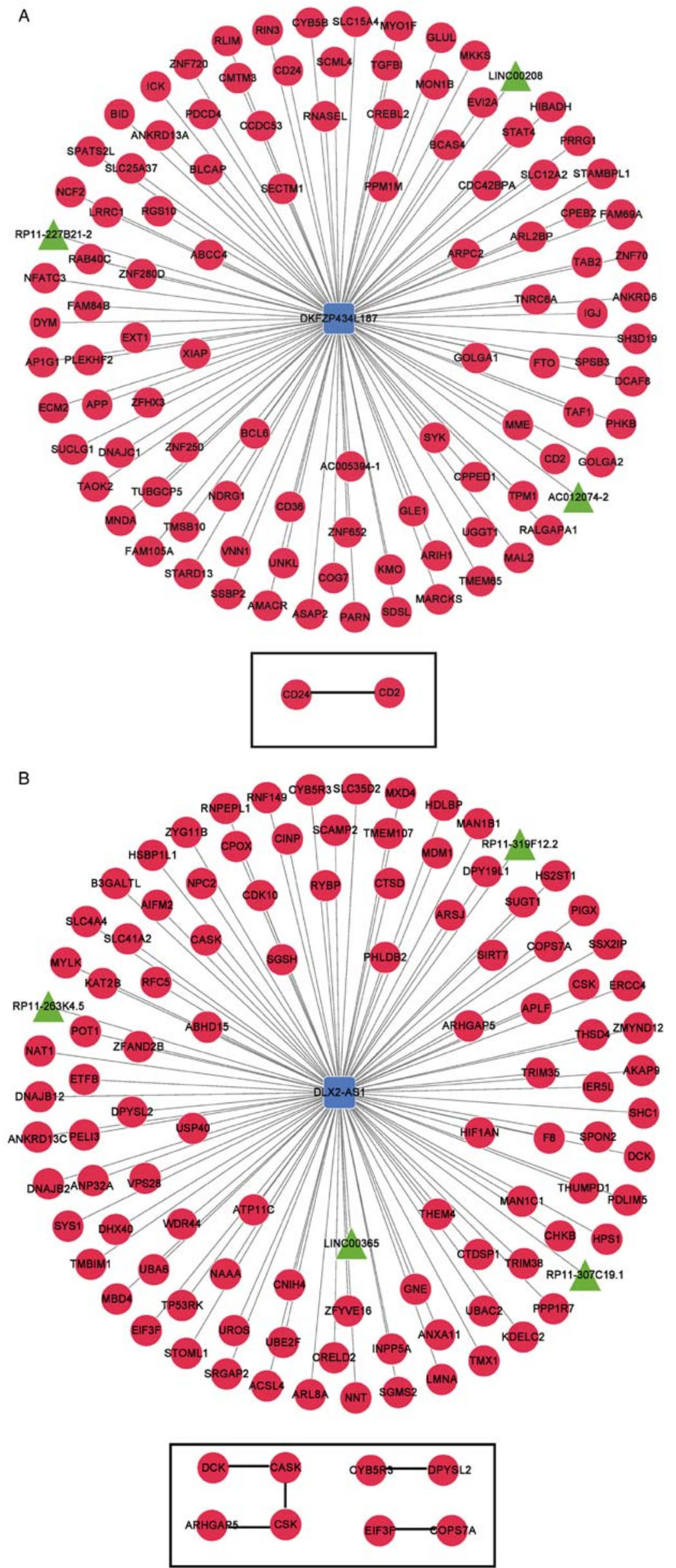

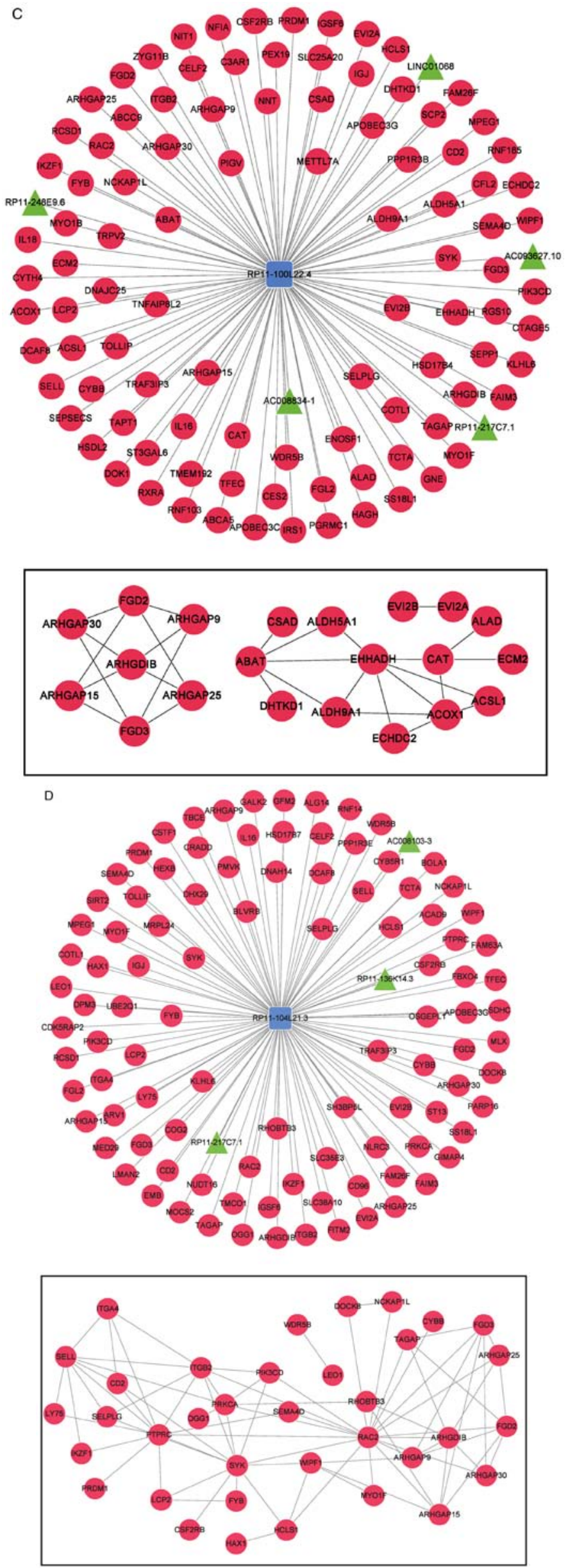

Figure 7. Continued. Top mRNAs co-expressed with (C) RP11-100L22.4 and (D) RP11-104L21.3. 

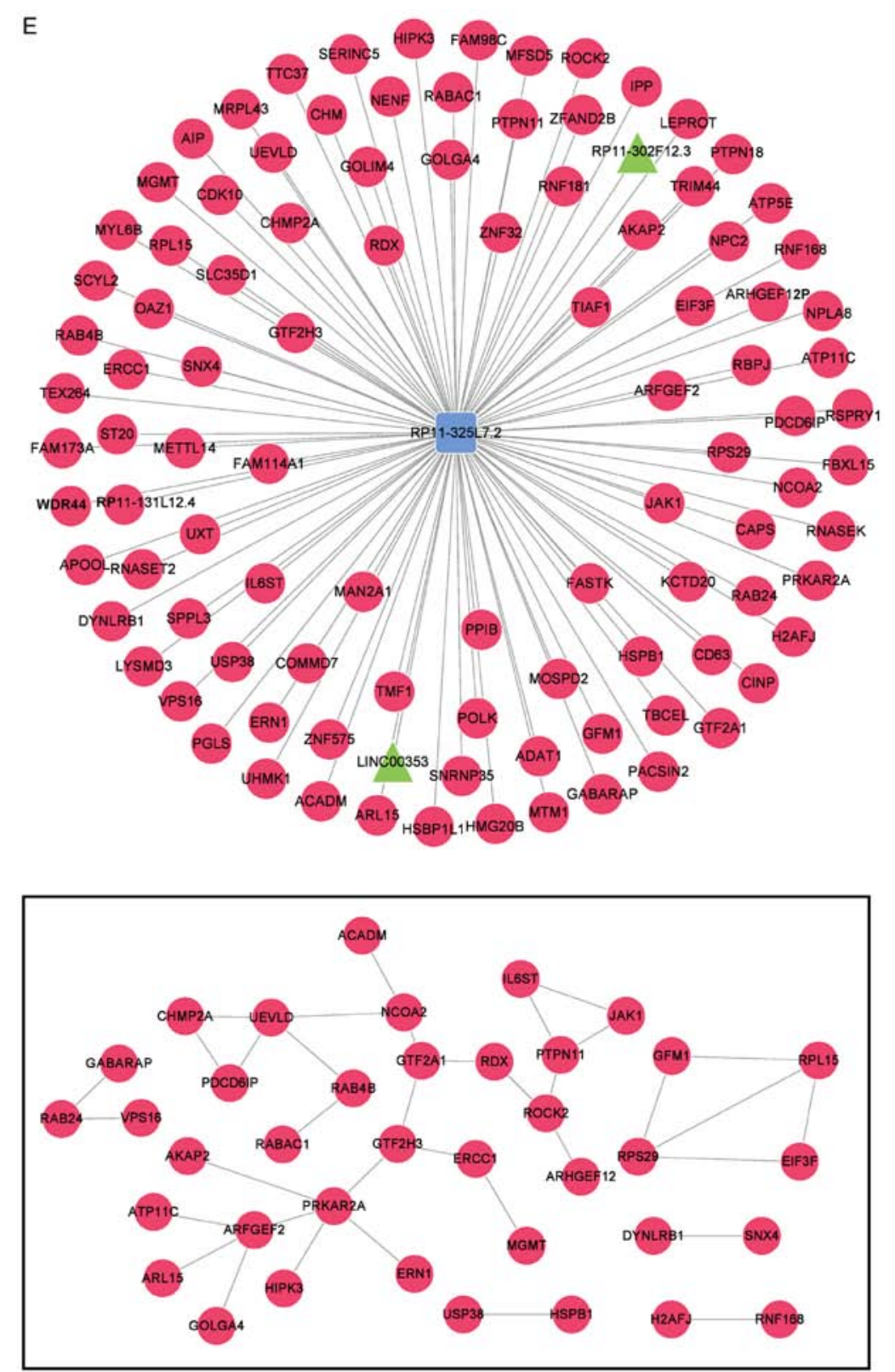

Figure 7. Continued. Top mRNAs co-expressed with (E) RP11-325L7.2 signature lncRNAs, and gene-gene co-expression networks of associated-mRNAs, are listed in this figure.

prognosis in HCC (25). In the present study, samples with high DLX2-AS1 expression had shorter survival times compared with those with low expression. The present results are inconsistent with previous studies, and further experiments are required to understand the discrepancy between the present and previous results.

The function of DKFZP434L187 (also termed LINC02249) is yet unknown. Based on the present results, this lncRNA may serve as a novel prognostic lncRNA biomarker for HCC. However, further studies are required in order to understand the role of this IncRNA in HCC.

It is known that lncRNAs participate in various biological processes such as transcription, translation, cellular differentiation, chromatin modification, regulation of gene expression, cell cycle and nuclear-cytoplasmic trafficking (26-28). IncRNAs guide chromatin-modifying complexes, thus allowing epigenetic modifications in cancer (29). According to the results presented in this study, WDR5B is a target gene of both RP11-100L22.4 and RP11-104L21.3. WDR5 is associated with the lncRNA HOXA distal transcript antisense RNA (HOTTIP), and its expression is associated with disease progression and predictive outcomes in HCC (30). HOTTIP binds directly to WDR5, and targets WDR5/mixed linear leukemia complexes across the HOXA gene cluster, thereby driving histone $\mathrm{H} 3$ lysine 4 trimethylation and gene transcription (31). Downregulated expression of HOX is also reported to be involved in the development of HCC (31). Therefore, RP11-100L22.4 and RP11-104L21.3 may participate in the prognosis of HCC through interactions with WDR5.

Lipid metabolism is an essential function of the liver, and any anomalies in this function may cause liver diseases, including fibrosis (32). Defects and/or deregulation of fatty acid metabolism have also been reported to be associated with HCC $(33,34)$. Fatty acid metabolism also serves an important 
role in determining the function of extra-mitochondrial pathways (35). In the present study, fatty acid metabolism was revealed as a potentially dysfunctional pathway associated with the signature lncRNA differentially expressed in the HCC samples studied. Thus, fatty acid metabolism may be a potential pathway influencing the prognosis of patients with HCC.

Although these predictive results are valuable, the present study has certain limitations. The expression of these lncRNAs should be experimentally validated. In addition, substantial experiments should be performed to confirm the prognostic accuracy of these five lncRNAs in HCC.

In conclusion, the signature lncRNAs screened in this study (RP11-325L7.2, DKFZP434L187, RP11-100L22.4, DLX2-AS1 and RP11-104L21.3) may serve as novel lncRNA biomarkers to predict the prognosis of HCC. Further studies of these lncRNAs and associated genes may contribute to a deeper understanding of the underlying mechanism of HCC development.

\section{Acknowledgements}

Not applicable.

\section{Funding}

The present study was supported by the Presidential Foundation of the 302 Military Hospital, China (grant no. YNKT2014027).

\section{Availability of data and materials}

The datasets used and/or analyzed during the current study are available from the corresponding author on reasonable request.

\section{Authors' contributions}

YQS analyzed the data and wrote the manuscript. LW, FFZ and XS collected and analyzed the data. JJ, FZ and SY drafted the manuscript and contributed to the experimental design. HHL conceived, designed the study and revised the manuscript. All authors read and approved the final manuscript.

\section{Ethics approval and consent to participate}

Not applicable.

\section{Patient consent for publication}

Not applicable.

\section{Competing interests}

The authors declare that they have no competing interests.

\section{References}

1. Forner A, Llovet JM and Bruix J: Hepatocellular carcinoma. Lancet 379: 1245-1255, 2012.

2. McGlynn KA and London WT: The global epidemiology of hepatocellular carcinoma: Present and future. Clin Liver Dis 15: 223-243, vii-x, 2011.
3. Yang Z, Zhou L, Wu LM, Lai MC, Xie HY, Zhang F and Zheng SS: Overexpression of long non-coding RNA HOTAIR predicts tumor recurrence in hepatocellular carcinoma patients following liver transplantation. Ann Surg Oncol 18: 1243-1250, 2011.

4. Llovet JM, Ricci S, Mazzaferro V, Hilgard P, Gane E, Blanc JF, de Oliveira AC, Santoro A, Raoul JL, Forner A, et al: Sorafenib in advanced hepatocellular carcinoma. N Engl J Med 359: 378-390, 2008 .

5. Zhou J, Li X, Wu M, Lin C, Guo Y and Tian B: Knockdown of long noncoding RNA GHET1 inhibits cell proliferation and invasion of colorectal cancer. Oncol Res 23: 303-309, 2016.

6. Yang F, Huo XS, Yuan SX, Zhang L, Zhou WP, Wang F and Sun SH: Repression of the long noncoding RNA-LET by histone deacetylase 3 contributes to hypoxia-mediated metastasis. Mol Cell 49: 1083-1096, 2013.

7. Zhu Y, Yu M, Li Z, Kong C, Bi J, Li J, Gao Z and Li Z: NcRAN, a newly identified long noncoding RNA, enhances human bladder tumor growth, invasion, and survival. Urology 77: 510.e1-5, 2011.

8. Prensner JR and Chinnaiyan AM: The emergence of lncRNAs in cancer biology. Cancer Discov 1: 391-407, 2011.

9. Wang F, Ying HQ, He BS, Pan YQ, Deng QW, Sun HL, Chen J, Liu X and Wang SK: Upregulated lncRNA-UCA1 contributes to progression of hepatocellular carcinoma through inhibition of miR-216b and activation of FGFR1/ERK signaling pathway. Oncotarget 6: 7899-7917, 2015.

10. Li T, Xie J, Shen C, Cheng D, Shi Y, Wu Z, Deng X, Chen H, Shen B, Peng C, et al: Upregulation of long noncoding RNA ZEB1-AS1 promotes tumor metastasis and predicts poor prognosis in hepatocellular carcinoma. Oncogene 35: 1575-1584, 2016.

11. Hua L, Wang CY, Yao KH, Chen JT, Zhang JJ and Ma WL: High expression of long non-coding RNA ANRIL is associated with poor prognosis in hepatocellular carcinoma. Int J Clin Exp Pathol 8: 3076-3082, 2015.

12. Ishibashi M, Kogo R, Shibata K, Sawada G, Takahashi Y, Kurashige J, Akiyoshi S, Sasaki S, Iwaya T, Sudo T, et al: Clinical significance of the expression of long non-coding RNA HOTAIR in primary hepatocellular carcinoma. Oncol Rep 29: 946-950, 2013.

13. Tu ZQ, Li RJ, Mei JZ and Li XH: Down-regulation of long non-coding RNA GAS5 is associated with the prognosis of hepatocellular carcinoma. Int J Clin Exp Pathol 7: 4303-4309, 2014.

14. Anders S and Huber W: Differential expression analysis for sequence count data. Genome Biol 11: R106, 2010.

15. Robinson MD, McCarthy DJ and Smyth GK: edgeR: A Bioconductor package for differential expression analysis of digital gene expression data. Bioinformatics 26: 139-140, 2010.

16. Wang P, Wang Y, Hang B, Zou X and Mao JH: A novel gene expression-based prognostic scoring system to predict survival in gastric cancer. Oncotarget 7: 55343-55351, 2016.

17. Hanley JA: The robustness of the 'binormal' assumptions used in fitting ROC curves. Med Decis Making 8: 197-203, 1988.

18. Adler P, Kolde R, Kull M, Tkachenko A, Peterson H, Reimand J and Vilo J: Mining for coexpression across hundreds of datasets using novel rank aggregation and visualization methods. Genome Biol 10: R139, 2009.

19. Harrow J, Frankish A, Gonzalez JM, Tapanari E, Diekhans M, Kokocinski F, Aken BL, Barrell D, Zadissa A, Searle S, et al: GENCODE: The reference human genome annotation for The ENCODE Project. Genome Res 22: 1760-1774, 2012.

20. Huang da W, Sherman BT and Lempicki RA: Systematic and integrative analysis of large gene lists using DAVID bioinformatics resources. Nat Protoc 4: 44-57, 2009.

21. Wu Y, Wang PS, Wang BG, Xu L, Fang WX, Che XF, Qu XJ, Liu YP and Li Z: Genomewide identification of a novel six-LncRNA signature to improve prognosis prediction in resectable hepatocellular carcinoma. Cancer Med 7: 6219-6233, 2018.

22. Wang J, Pu J, Yao T, Lu X and Deng Y: Four long noncoding RNAs as potential prognostic biomarkers for hepatocellular carcinoma. J Cell Physiol 234: 8709-8716, 2018

23. Abd El Gwad A, Matboli M, El-Tawdi A, Habib EK, Shehata H, Ibrahim D and Tash F: Role of exosomal competing endogenous RNA in patients with hepatocellular carcinoma. J Cell Biochem 119: 8600-8610, 2018.

24. Fang T, Fang Y, Xu X, He M, Zhao Z, Huang P, Yuan F, Guo M, Yang B and Xia J: Actinidia chinensis Planch root extract attenuates proliferation and metastasis of hepatocellular carcinoma by inhibiting epithelial-mesenchymal transition. J Ethnopharmacol 231: 474-485, 2019. 
25. Liu J, Cui X, Qu L, Hua L, Wu M, Shen Z, Lu C and Ni R: Overexpression of DLX2 is associated with poor prognosis and sorafenib resistance in hepatocellular carcinoma. Exp Mol Pathol 101: 58-65, 2016.

26. Kim ED and Sung S: Long noncoding RNA: Unveiling hidden layer of gene regulatory networks. Trends Plant Sci 17: 16-21, 2012

27. Yoon JH, Abdelmohsen $\mathrm{K}$ and Gorospe M: Posttranscriptional gene regulation by long noncoding RNA. J Mol Biol 425: 3723-3730, 2013.

28. Wilusz JE, Sunwoo H and Spector DL: Long noncoding RNAs: Functional surprises from the RNA world. Genes Dev 23: 1494-1504, 2009.

29. Harries LW: Long non-coding RNAs and human disease. Biochem Soc Trans 40: 902-906, 2012.

30. Quagliata L, Matter MS, Piscuoglio S, Arabi L, Ruiz C, Procino A, Kovac M, Moretti F, Makowska Z, Boldanova T, et al: Long noncoding RNA HOTTIP/HOXA13 expression is associated with disease progression and predicts outcome in hepatocellular carcinoma patients. Hepatology 59: 911-923, 2014.

31. Wang KC, Yang YW, Liu B, Sanyal A, Corces-Zimmerman R, Chen Y, Lajoie BR, Protacio A, Flynn RA, Gupta RA, et al: A long noncoding RNA maintains active chromatin to coordinate homeotic gene expression. Nature 472: 120-124, 2011.
32. Moustafa T, Fickert P, Magnes C, Guelly C, Thueringer A, Frank S, Kratky D, Sattler W, Reicher H, Sinner F, et al: Alterations in lipid metabolism mediate inflammation, fibrosis, and proliferation in a mouse model of chronic cholestatic liver injury. Gastroenterology 142: 140-151, e12, 2012.

33. Li Z, Guan M, Lin Y, Cui X, Zhang Y, Zhao Z and Zhu J: Aberrant lipid metabolism in hepatocellular carcinoma revealed by liver lipidomics. Int J Mol Sci 18: 2550, 2017.

34. Zhou L, Wang Q, Yin P, Xing W, Wu Z, Chen S, Lu X, Zhang Y, Lin $\mathrm{X}$ and $\mathrm{Xu} \mathrm{G}$ : Serum metabolomics reveals the deregulation of fatty acids metabolism in hepatocellular carcinoma and chronic liver diseases. Anal Bioanal Chem 403: 203-213, 2012.

35. Ockner RK, Kaikaus RM and Bass NM: Fatty-acid metabolism and the pathogenesis of hepatocellular carcinoma: Review and hypothesis. Hepatology 18: 669-676, 1993.

(i) (9) This work is licensed under a Creative Common Attribution-NonCommercial-NoDerivatives 4.0 International (CC BY-NC-ND 4.0) License. 